\title{
Emergence of Feature-Specific Connectivity in Cortical Microcircuits in the Absence of Visual Experience
}

\author{
(D)Ho Ko, ${ }^{1}$ Thomas D. Mrsic-Flogel, ${ }^{1,2}$ and Sonja B. Hofer ${ }^{1,2}$ \\ ${ }^{1}$ Department of Neuroscience, Physiology and Pharmacology, University College London, London, WC1E 6DE, United Kingdom, and ${ }^{2}$ Biozentrum, \\ University of Basel, 4056 Basel, Switzerland
}

In primary visual cortex (V1), connectivity between layer $2 / 3(\mathrm{~L} 2 / 3)$ excitatory neurons undergoes extensive reorganization after the onset of visual experience whereby neurons with similar feature selectivity form functional microcircuits (Ko et al., 2011, 2013). It remains unknown whether visual experience is required for the developmental refinement of intracortical circuitry or whether this maturation is guided intrinsically. Here, we correlated the connectivity between V1 L2/3 neurons assayed by simultaneous whole-cell recordings in vitro to their response properties measured by two-photon calcium imaging in vivo in dark-reared mice. We found that neurons with similar responses to oriented gratings or natural movies became preferentially connected in the absence of visual experience. However, the relationship between connectivity and similarity of visual responses to natural movies was not as strong in dark-reared as in normally reared mice. Moreover, dark rearing prevented the normally occurring loss of connections between visually nonresponsive neurons after eye opening (Ko et al., 2013). Therefore, our data suggest that the absence of visual input does not prevent the emergence of functionally specific recurrent connectivity in cortical circuits; however, visual experience is required for complete microcircuit maturation.

Key words: experience-dependent; functional microcircuit organization; microcircuit development; mouse visual cortex; synaptic connectivity; two-photon imaging

\section{Introduction}

Many aspects of the organization and function of the visual system develop in the absence of visual experience. Neurons in primary visual cortex (V1) are feature selective in visually naive animals (Hubel and Wiesel, 1963; Blakemore and Van Sluyters, 1975; Frégnac and Imbert, 1978; Chapman and Stryker, 1993; Krug et al., 2001; White et al., 2001; Rochefort et al., 2011; Ko et al., 2013; Sarnaik et al., 2014), and in mouse V1 the spatial structure of receptive fields is mature already at eye opening (Ko et al., 2013), suggesting that the feedforward circuitry is largely established before the onset of vision. However, some visual response properties mature after eye opening, and several aspects of the visual circuitry require visual input to develop or refine (Hubel and Wiesel, 1963; Blakemore and Van Sluyters, 1975; Frégnac and Imbert, 1978; Chapman and Stryker, 1993; Li et al., 2006; Rochefort et al., 2011; Ko et al., 2013). In the visual cortex, dark rearing alters the maturation of both inhibitory circuits and longrange excitatory projections (White et al., 2001; Morales et al.,

\footnotetext{
Received March 4, 2014; revised May 8, 2014; accepted June 13, 2014.

Author contributions: H.K., T.D.M.-F., and S.B.H. designed research;H.K. and S.B.H. performed research; H.K. and S.B.H. analyzed data; H.K., T.D.M.-F., and S.B.H. wrote the paper.

This work is supported by the Wellcome Trust (T.D.M.-F., S.B.H.), the European Research Council (T.D.M.-F., S.B.H.), and the UCL Excellence Fellowship Programme (S.B.H.). We thank Claudia Clopath for helpful discussions. The authors declare no competing financial interests.

Correspondence should be addressed to Dr. Sonja B. Hofer, Biozentrum, University of Basel, Klingelbergstrasse 50/70, 4056 Basel, Switzerland. E-mail: sonja.hofer@unibas.ch.

H. Ko's present address: Faculty of Medicine, Chinese University of Hong Kong, Shatin, New Territories, Hong Kong.

DOI:10.1523/JNEUROSCI.0875-14.2014

Copyright $\odot 2014$ the authors $\quad 0270-6474 / 14 / 349812-05 \$ 15.00 / 0$
}

2002; Chattopadhyaya et al., 2004; Katagiri et al., 2007). Therefore, it might be expected that the functional maturation of local excitatory circuits in the cortex also depends on visual experience, but this remains unexplored.

We recently found that, after the onset of vision, local cortical connections between L2/3 excitatory neurons reorganize, such that cells with similar responses to visual features become progressively more connected (Ko et al., 2013). However, it is unknown whether visual input is crucial for this reorganization of recurrent connectivity or whether it is instead guided by intrinsic, experience-independent factors. In this study, we tested the role of visual experience in the maturation of local excitatory circuits by relating the synaptic connectivity of L2/3 pyramidal neurons determined in vitro to their response properties characterized by twophoton calcium imaging in vivo in dark-reared mice. We found that intrinsic factors and visual experience seem to jointly govern the maturation of functional microcircuits in visual cortex.

\section{Materials and Methods}

In vivo two-photon calcium imaging. All experimental procedures were licensed and performed in accordance with institutional and national animal welfare guidelines. Experiments were performed on C57BL/6 mice of either sex, aged postnatal day (P) 13-14 reared normally (12 h light/dark cycle), and 22-26 reared normally or reared in complete darkness from shortly before eye opening (P11-P12) until P21-P25. Anesthesia, surgery, loading of Oregon Green BAPTA-1 AM (Invitrogen), and two-photon calcium imaging were performed as described previously (Hofer et al., 2011; Ko et al., 2011, 2013). Visually evoked responses from all neurons in a small cortical volume in monocular V1 were obtained at sequential depths at $7.6 \mathrm{~Hz}$ imaging rate while displaying visual stimuli generated using MATLAB Psychophysics Toolbox (MathWorks) (Brain- 
ard, 1997) on an LCD monitor (60 Hz refresh rate) as previously described (Hofer et al., 2011; Ko et al., 2011, 2013). To measure the orientation preference and selectivity of neurons, square-wave gratings ( 0.035 cycles/degree, 2 cycles/s, $100 \%$ contrast) drifted in eight different directions (randomly interleaved), with the grating standing for 1.4-1.9 $\mathrm{s}$ before moving for $0.9-1.5 \mathrm{~s}$ (six repetitions). Natural movies consisted of 30-40 s sequences of moving scenes recorded from a camera headmounted on a mouse or cat and compilations of David Attenborough's Life of Mammals (BBC) (adjusted to 70\% mean contrast, continuously looped 6 times).

Data analysis. Image processing and extraction of calcium signals were performed using custom-written software in MATLAB and LabVIEW as previously described (Ko et al., 2011, 2013), and spike trains were inferred from calcium signals using a fast non-negative deconvolution method (Vogelstein et al., 2010, Hofer et al., 2011).

Neurons responsive to drifting gratings were identified by one-way ANOVA $(p<0.05)$. The mean of inferred firing rates during grating drift was taken as the response to each stimulus. From each trial, we obtained one orientation tuning curve, and neurons were defined as reliably responsive if the mean cross-correlation between all pairs of curves was $>$ 0.1 . Responses were then averaged to obtain the average orientation tuning curve for a given neuron. This curve was Fourier interpolated to 360 points, from which the preferred direction $\left(R_{\text {pref }}\right)$ and the average of the orthogonal directions $\left(R_{\text {ortho }}\right)$ were determined. Orientation selectivity index (OSI) was calculated as follows: $\left(R_{\text {pref }}-R_{\text {ortho }}\right) /\left(R_{\text {pref }}+R_{\text {ortho }}\right)$.

Responses to natural movies were moving-average filtered (3-frame window), and the corresponding frames during stimulus presentation were treated as groups and tested for differences by one-way ANOVA. Neurons with $p$ values $<1 \times 10^{-4}$ were considered visually responsive. For further analysis, nonfiltered traces were used. We calculated Pearson's correlation coefficient of average responses to the movies (peristimulus time histograms) as the signal correlation, of the entire response sequence as response correlation between cell pairs, and between responses in individual trials of one cell as a measure of reliability of natural-movie evoked responses.

In vitro whole-cell recordings of neurons functionally characterized in vivo. In vitro recordings after in vivo calcium imaging were performed as described previously (Ko et al., 2011, 2013). In short, after in vivo twophoton calcium imaging of visual responses, the mouse brain was rapidly removed and dissected in ice-cold artificial CSF. The imaged region in the coronally sliced cortex was identified by the presence of red fluorescent microspheres injected in vivo, and whole-cell recordings from two to six neurons were performed in the imaged region. The presence of synaptic connections was tested by evoking five spikes at $30 \mathrm{~Hz}$ in each cell, repeated 30-120 times. We performed 3D image registration of in vivo and in vitro image stacks by affine transformation using custom-written MATLAB software subsequent to the experiment to match neurons patched in vitro and imaged in vivo (Ko et al., 2011).

Connection probabilities were calculated as the number of connections detected over the number of potential connections assayed. To relate connectivity to functional properties, the asymptotic CochranArmitage test for trend was used to test for significance of linear trends (Agresti, 2002). Only neuronal pairs in which both neurons were located at $>60 \mu \mathrm{m}$ depth from the slice surface and with an intersoma distance of $<50 \mu \mathrm{m}$ (mean distance \pm SD: P13-P15, $24 \pm 9 \mu \mathrm{m}, \mathrm{P} 22-\mathrm{P} 26,25 \pm 10$ $\mu \mathrm{m}$, dark-reared, $26 \pm 11 \mu \mathrm{m}$ ) were included in the analysis.

\section{Results}

\section{Orientation-specific connectivity matures without visual experience}

To investigate the role of visual experience in shaping visual responses and the functional specificity of local synaptic connections, we performed experiments in mice reared in complete darkness from shortly before eye opening (P11-P12) until P21$\mathrm{P} 25$. Data from these experiments were compared with previously obtained data from normally reared mice at eye opening (P13-P14) and P22-P26 (Ko et al., 2013). We used in vivo twophoton calcium imaging to characterize visual response proper- ties of neurons in L2/3 of monocular mouse V1. We observed significantly lower proportions of reliably responsive and orientation-selective neurons at eye opening than in more mature mice but no difference between animals reared normally and in the dark (mean proportion \pm SEM, P13-P14, $31.6 \pm 5.2 \%$, $n=13$; P22-P26, $54.5 \pm 2.4 \%, n=18$; dark-reared, $49.6 \pm 2.6 \%$, $n=6, p=2 \times 10^{-4}$, one-way ANOVA test; P13-P14 vs P22-P26, $p<1 \times 10^{-3}, \mathrm{P} 13-\mathrm{P} 14$ vs dark-reared, $p=0.027, \mathrm{P} 22-\mathrm{P} 26$ vs dark-reared, $p=0.727$, Tukey's HSD post hoc test). Equally, the degree of orientation selectivity among responsive neurons, quantified by the OSI, was similar in normally and dark-reared mice, and slightly but significantly lower at eye opening (Fig. $1 A$; mean OSI of imaged planes \pm SD: P13-P14, $0.61 \pm 0.09, n=90$ imaged planes from 13 mice P22-P26 normally reared, $0.66 \pm$ $0.05, n=125$ imaged planes from 18 mice; dark-reared, $0.68 \pm$ $0.05, n=48$ imaged planes from 6 mice; P13-P14 vs P22-P26, $p=7.0 \times 10^{-8}, \mathrm{P} 13-\mathrm{P} 14$ vs dark-reared, $p=8.3 \times 10^{-7}, \mathrm{P} 22-$ $\mathrm{P} 26$ vs dark-reared, $p=0.23$, rank sum test). Therefore, similar to a previous study in mouse V1 (Rochefort et al., 2011), we found that orientation-selective responses emerge early and do not require visual experience for their maturation. However, the variability of neuronal responses to the preferred grating direction, although lower than at eye opening, was slightly higher in dark-reared than in normally reared mice (Fig. $1 B$; mean coefficient of variation $\pm \mathrm{SD}$ : $\mathrm{P} 13-\mathrm{P} 14,0.95 \pm 0.17, n=90$ imaged planes; P22-P26, $0.71 \pm 0.10$, $n=125$; dark-reared, $0.77 \pm 0.12, n=48$; $\mathrm{P} 13-\mathrm{P} 14$ vs $\mathrm{P} 22-\mathrm{P} 26, p=$ $4.0 \times 10^{-22}, \mathrm{P} 13-\mathrm{P} 14$ vs dark-reared, $p=3.0 \times 10^{-9}, \mathrm{P} 22-\mathrm{P} 26$ vs dark-reared, $p=0.0087$, rank sum test).

After characterizing visual response properties of all neurons in a volume of $\sim 285 \times 285 \times 40-120 \mu^{3}$ in L2/3, we then performed simultaneous multiple whole-cell recordings in the same tissue in vitro and assessed connectivity between up to six pyramidal neurons whose responses had been characterized in vivo (Fig. $1 C-F$ ). The overall connection probability between neighboring L $2 / 3$ cells (cortical distance $<50 \mu \mathrm{m}$ ) was similar at eye opening and in more mature mice with or without visual experience (Fig. 1G; P13-P14, 58 of 353, 16.4\%; P22-P26, 64 of 295, 21.7\%; dark-reared, 44 of 233, 18.9\%, $p=0.23, \chi^{2}$ test). We then identified the cells recorded in vitro in the in vivo image stacks (Ko et al., 2011), which allowed us to relate the probability of finding a connection between pyramidal cell pairs in vitro to their orientation preference. As previously reported, we found no relationship between the similarity in preferred orientation of pairs of neurons and their connection probability at eye opening (Fig. $1 H$; P13-P14, $p=0.27$, Cochran-Armitage test), whereas in more mature animals neurons tuned to similar orientations became preferentially connected (Fig. $1 H$; P22-P26, $p=0.034$ ). In mice reared in the dark, the same relationship emerged, such that pairs of cells preferring similar orientations were more than twice as likely to be connected than cell pairs with orthogonal orientation preference (Fig. $1 H$; dark-reared, $p=0.028$ ). These data indicate that the emergence of orientation-specific connectivity is largely independent of visual experience.

\section{Connectivity specific for response similarity to natural movies partly matures in dark-reared mice}

In addition to orientation selectivity, we also analyzed responses of neurons in L2/3 to natural movie sequences (for details, see Materials and Methods). The fraction of neurons significantly responsive to the natural movie were similar at P13-P14, and in normally and dark-reared P22-P26 mice (Fig. 2A; mean proportion \pm SEM, P13-P14, $50.1 \pm 5.7 \%, n=13$; P22-P26, $42.6 \pm$ $4.1 \%, n=18$; dark-reared, $49.1 \pm 6.0 \%, n=6, p=0.49$, one-way 

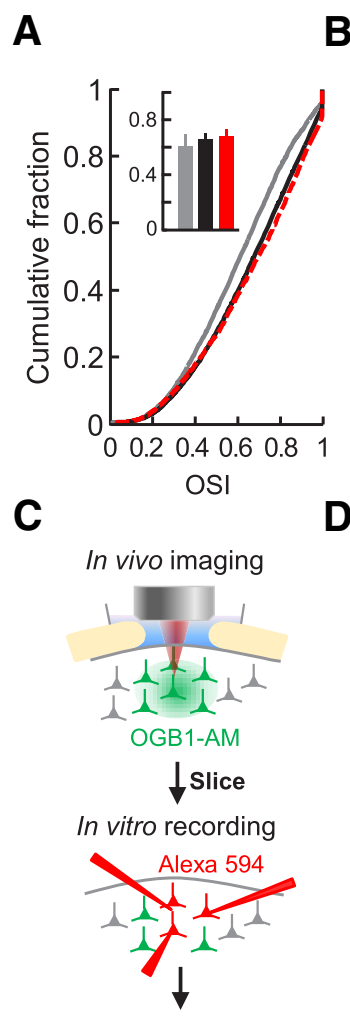

Match cells in vitro and in vivo

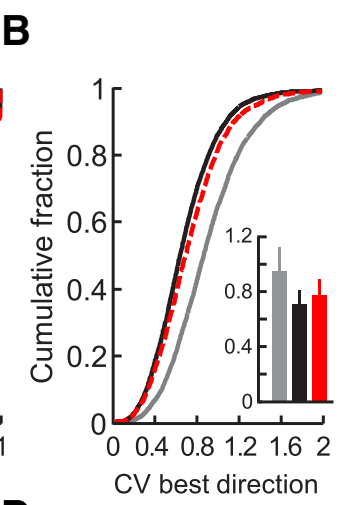

D

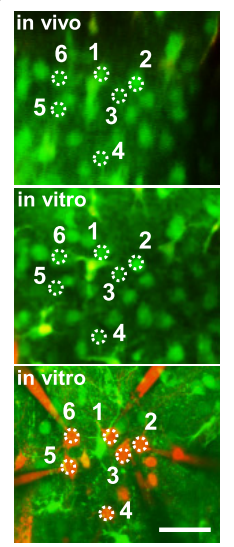

E

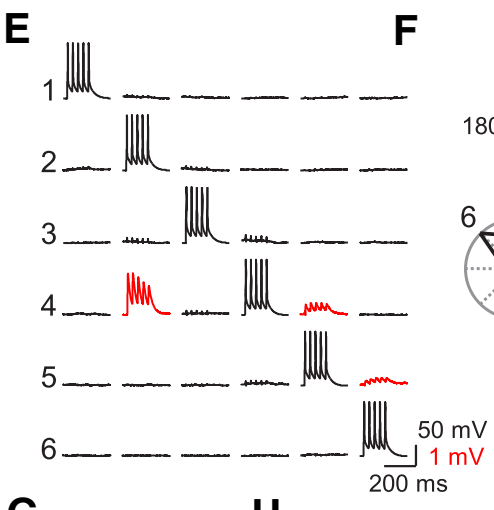

H

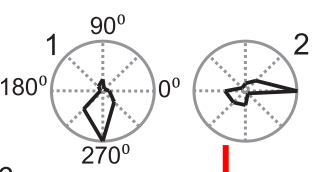

G

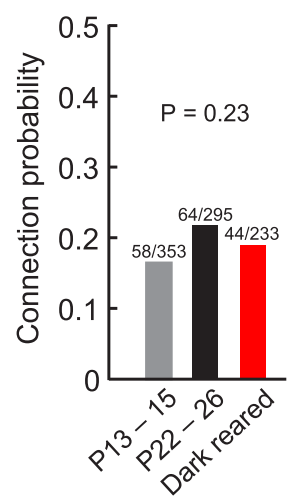

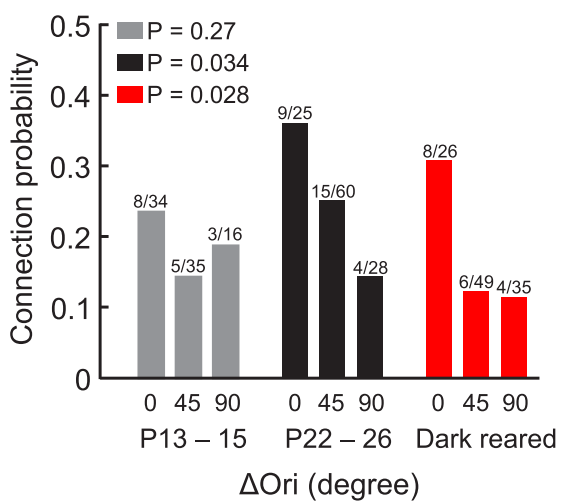

Figure 1. Visual response properties and connectivity of $\mathrm{L} 2 / 3$ pyramidal cells after dark rearing. $A, B$, Cumulative distributions of orientation selectivity index (OSI) for visually responsive neurons $(\boldsymbol{A})$ and of the coefficient of variation (CV) of responses to the preferred grating direction (B) at P13-P15 (gray), P22-P26 (black), and after dark rearing (red, dashed line). Insets, Average and SD of means of OSI and CV of all imaged planes (number of planes: P13-P15, $n=90 ;$ P22-P26, $n=125$; dark-reared, $n=48$ ). C, Schematic showing the experimental design. D, Example sextuplet of neurons shown in a transformed in vivo image (top), in a brain slice (middle), and during whole-cell recordings (bottom). Scale bar, $30 \mu \mathrm{m}$. $\boldsymbol{E}$, Membrane potential recordings from neurons shown in $\boldsymbol{D}$. Evoked spikes in one cell and average traces of related postsynaptic potentials in the other cells are shown in each column. Traces indicating synaptic connections are colored in red. Some traces show stimulus artifacts clearly distinguishable from EPSPs. $\boldsymbol{F}$, Polar plots showing normalized response strength to different grating directions from the cells in $\boldsymbol{D}$ and $\boldsymbol{E}$. Synaptic connections between the neurons are indicated by red arrows. G, Overall connection probability between excitatory L2/3 neurons at eye opening, and in more mature normally and dark-reared animals (Chi-squared test). $\boldsymbol{H}$, Relationship between connection probability and difference in preferred orientation ( $\Delta$ Ori) for responsive and orientation selective pairs $(0 \mathrm{SI}>0.4)(\mathrm{Cochran}-$ Armitage test). P13-P15, $n=13$ mice; P22-P26, $n=18$ mice; dark-reared, $n=6$ mice. Numbers above bars denote number of observed connections out of connections sampled.

ANOVA test). Among responsive neurons, reliability of natural movie responses in dark-reared mice was significantly higher than at eye opening but lower than in normally reared mice of the same age (Fig. 2B; mean average trial-to-trial correlation of imaged planes \pm SD: P13-P14, $0.070 \pm 0.021, n=90 ;$ P22-P26, $0.107 \pm 0.027, n=125$; dark-reared, $0.089 \pm 0.024, n=48$; P13-P14 vs P22-P26, $p=4.5 \times 10^{-19}$, P13-P14 vs dark-reared, $p=1.6 \times 10^{-5}, \mathrm{P} 22-\mathrm{P} 26$ vs dark-reared, $p=6.7 \times 10^{-5}$, rank sum test). Therefore, reliability of responses to naturalistic visual stimuli increased during development in the dark but did not reach the level found in normally reared animals.

We then explored the relationship between connection probability and similarity of responses to the natural movie by computing signal correlations between cell pairs (correlation coefficient between peristimulus time histograms of two cells). We have previously shown that connectivity among L2/3 pyramidal neurons undergoes refinement after eye opening, whereby a much steeper relationship between connection probability and signal correlation becomes apparent in more mature V1 (Fig. 2C; P13-P14, $p=0.100$, P22-P26, $p=2 \times 10^{-3}$, Cochran-Armitage test). We also found a significant relationship in dark-reared mice, whereby pairs of neurons with very similar responses (signal correlation $\geq 0.15$ ) were more than twice as likely to be connected than pairs responding more differently to the natural movie (signal correlation $\leq 0.05$; Fig. $2 C$; dark-reared, $p=$ 0.026). However, the relationship was not as steep as in normally reared mice of the same age. Specifically, there were more connections between neurons with signal correlations $>0.05$ when mice were reared normally compared with mice without visual experience (Fig. 2C).

\section{Visual experience is required for the loss of connections between visually unresponsive neurons}

Next, we tested whether the previously observed loss of connections between visually unresponsive neurons is experiencedependent (Ko et al., 2013). At eye opening, neurons that were not reliably responsive to visual stimuli were connected to each other as frequently as visually driven neurons (Fig. 2D; P13-P14, $p=0.21, \chi^{2}$ test). In contrast, in normally reared mature mice the connection probability of nonresponsive neurons was more than four times lower than that of visually responsive cells (Fig. 2D; P22-P26, $p=2.8 \times 10^{-5}$ ). This developmental loss of connections did not occur in the absence of visual experience because responsive and nonresponsive neurons remained similarly connected in dark-reared mice (Fig. $2 D$; dark-reared, $p=0.91$ ).

During development, activity of L2/3 pyramidal neurons becomes less correlated (Rochefort et al., 2009; Ko et al., 2013) (Fig. $2 E, F)$. Response correlations between visually responsive neu- 

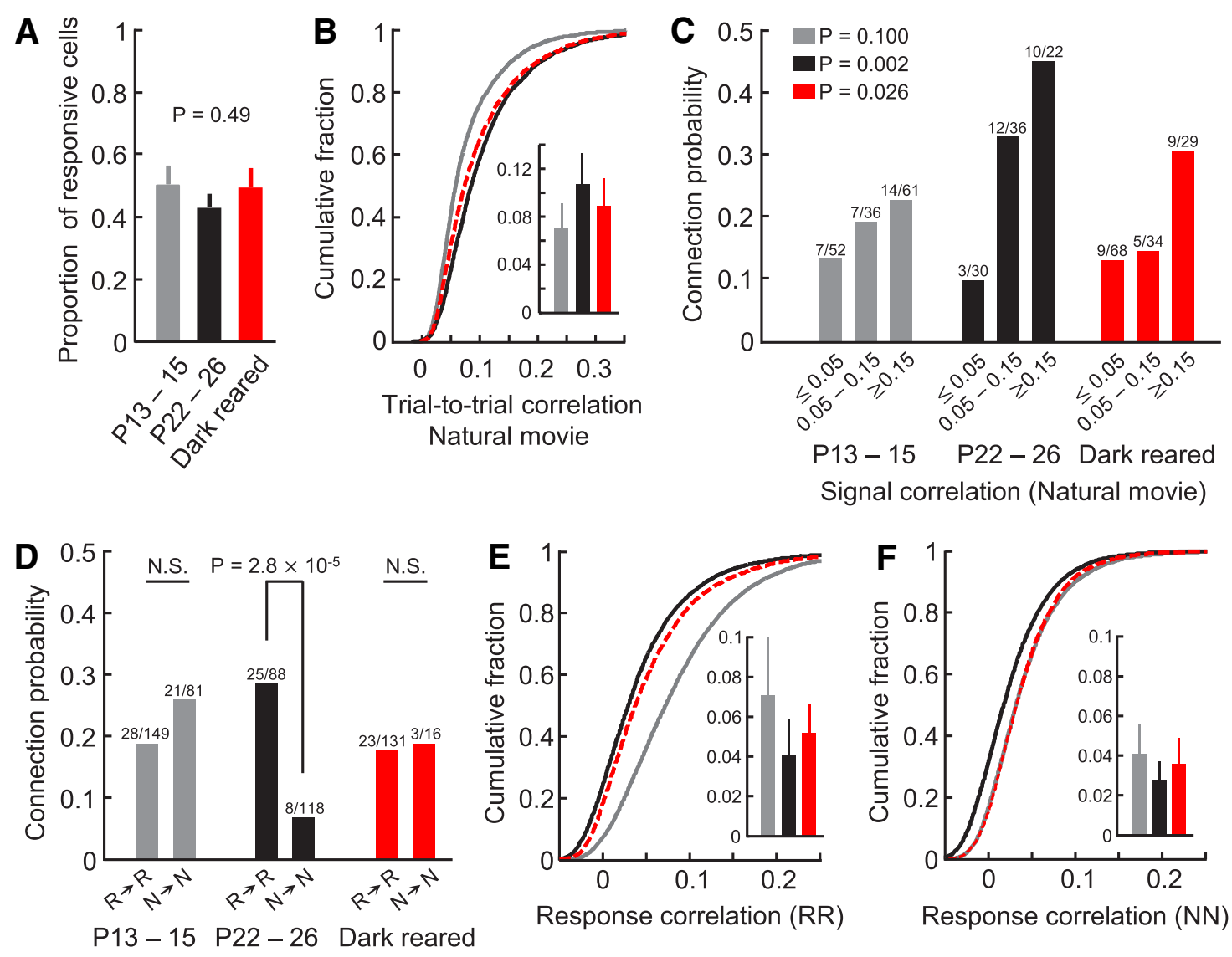

Figure 2. Relationship between responses to natural movies and connectivity after dark rearing. $A$, Proportions of $L 2 / 3$ neurons significantly responsive to the natural movie at eye opening and in more mature normally and dark-reared mice. B, Cumulative distribution of average trial-to-trial correlation of responses to the natural movie at P13-P15 (gray), P22-P26 (black), and after dark rearing (red, dashed line). Inset, Average and SD of the means of all imaged planes of the three groups (number of planes: P13-P15, $n=90 ; P 22-P 26, n=125 ;$ dark-reared, $n=48$ ). C, Relationship between connection probability and signal correlation between pairs of neurons significantly responsive to the natural movie (Cochran-Armitage test). $D$, Connection probability of neurons significantly responsive to the natural movie $(R \rightarrow R)$ and between nonresponsive neurons $(N \rightarrow N)\left(\chi^{2}\right.$ test). N.S., Not significant. $\boldsymbol{E}$, $\boldsymbol{F}$, Cumulative distributions of time-varied response correlation coefficients during the natural movie between visually responsive $(\boldsymbol{E})$, and nonresponsive cell pairs $(\boldsymbol{F})$. Insets, Average and SD of means of all imaged planes.

rons strongly decreased after eye opening regardless of visual experience, and to a slightly lesser extent in dark-reared animals (Fig. 2E; mean response correlation $\pm \mathrm{SD}, \mathrm{P} 13-\mathrm{P} 14,0.071 \pm$ $0.030, n=90$ imaged planes; P22-P26, $0.041 \pm 0.018, n=125$; dark-reared, $0.052 \pm 0.015, n=48$; P13-P14 vs P22-P26, $p=$ $1.5 \times 10^{-13}, \mathrm{P} 13-\mathrm{P} 14$ vs dark-reared, $p=4.2 \times 10^{-4}, \mathrm{P} 22-\mathrm{P} 26$ vs dark-reared, $p=2.3 \times 10^{-5}$, rank sum test). Interestingly, however, response correlations between visually nonresponsive neurons decreased only in normally reared animals. Activity correlations of nonresponsive neurons in dark-reared V1 remained as high as in $\mathrm{V} 1$ at eye opening (Fig. 2F; mean response correlation of imaged planes $\pm \mathrm{SD}, \mathrm{P} 13-\mathrm{P} 14,0.041 \pm 0.016, n=90$; $\mathrm{P} 22-\mathrm{P} 26,0.028 \pm 0.010, n=125$; dark-reared, $0.036 \pm 0.014$, $n=48$; P13-P14 vs P22-P26, $p=1.0 \times 10^{-9}, \mathrm{P} 13-\mathrm{P} 14$ vs darkreared, $p=0.25$, P22-P26 vs dark-reared, $p=4.0 \times 10^{-4}$, rank sum test). In dark-reared mice, therefore, the presence of increased activity correlations in visually nonresponsive cell pairs parallels their increased connectivity (Fig. 2D).

\section{Discussion}

Synaptic connectivity in mature cortical circuits is highly nonrandom. In visual cortex, excitatory neurons preferentially connect to each other if they respond similarly to sensory input (Yoshimura et al., 2005; Ko et al., 2011). During development, feedforward input is established first, while functionally specific local circuits in L2/3 emerge subsequently, mainly after eye open- ing, whereby neurons with similar visual responses preferentially form recurrent connections (Ko et al., 2013). We found that rearing mice in darkness had a surprisingly moderate effect on the development of these functional microcircuits between visually responsive neurons. The relationship between connection probability and orientation preference was as steep in dark-reared as in normally reared animals. When relating connectivity to more complex response properties, however, such as the response to a dynamic, more naturalistic stimulus, we found that the refinement of connectivity between L2/3 pyramidal neurons was at least partly dependent on visual experience; although the relationship between connection probability and signal correlation in response to a naturalistic movie was steeper in dark-reared animals than at eye opening, it was even steeper in normally reared animals of the same age.

Nevertheless, the refinement of intracortical visual circuitry seems to a large degree to occur without visual input. What could be the mechanism of this maturation? Although the wave-like activity in the retina diminishes after eye opening independent of visual experience, retinal neurons remain highly spontaneously active in the dark (Demas et al., 2003). In mouse visual cortex at eye opening, L2/3 neurons exhibit a spatial receptive field structure that is indistinguishable from that in mature V1 (Ko et al., 2013). Because the feedforward inputs onto L $2 / 3$ cells therefore appear to be established early, cortical neurons may still receive 
patterned input driven by spontaneous activity originating in the retina and/or the lateral geniculate nucleus (Fiser et al., 2004; Ackman et al., 2012). Provided they share common feedforward input, activity-dependent synaptic plasticity could sculpt the reciprocal connections between L2/3 pyramidal cells, even in the absence of visual drive, as previously demonstrated in developmental models based on spike-timing-dependent plasticity (Clopath et al., 2010; Ko et al., 2013). Alternatively or in addition, other intrinsic mechanisms, such as the preferential electrical coupling between clonally related neurons, might be involved in microcircuit development (Li et al., 2012; Ohtsuki et al., 2012; Yu et al., 2012, Ko et al., 2013). Although our data indicate that the specific pattern and/or strong feedforward drive of normal visual input is necessary for a complete refinement of functional microcircuits, we cannot rule out that dark rearing only delays and not completely prevents the full maturation of local functional connectivity over longer time periods.

One clear effect of dark rearing was the maintenance of connections among neurons not significantly responsive to visual stimulation, whereas in normally reared animals connections between nonresponsive cells were lost after eye opening. This pruning of connections during normal development was predicted by the spike-timing-dependent plasticity models (Clopath et al., 2010; Ko et al., 2013), whereby cells not reliably activated by patterned input lose connections. It is conceivable that, in the absence of visual input during dark rearing, these cells remain more coactive with the local network and with each other. This is supported by the observation that the strength of response correlations between visually nonresponsive cells remained higher in mice reared in the dark, similar to immature mice at eye opening. The more correlated activity of nonresponsive cells in darkreared animals could prevent the loss of connections between them. This interpretation has to be taken with caution because the higher connectivity rate between visually nonresponsive neurons might itself be the cause of higher response correlations in V1 of dark-reared mice. This scenario is more unlikely, however, because the connection probability between nonresponsive cells is nevertheless low, and connections between $\mathrm{L} 2 / 3$ pyramidal cells are, on average, weak $(0.18 \mathrm{mV})$.

In conclusion, although visual experience is important for some aspects of the refinement of L2/3 microcircuits, the formation of functionally specific excitatory connections between visually responsive neurons can to a large extent be accomplished by intrinsic factors independent of visual input, most likely by the spontaneously generated activity in the retina or the thalamus acting on the template of preestablished feedforward connections.

\section{References}

Ackman JB, Burbridge TJ, Crair MC (2012) Retinal waves coordinate patterned activity throughout the developing visual system. Nature 490:219225. CrossRef Medline

Agresti A (2002) Categorical data analysis. New York: Wiley-Interscience.

Blakemore C, Van Sluyters RC (1975) Innate and environmental factors in the development of the kitten's visual cortex. J Physiol 248:663-716. Medline

Brainard DH (1997) The Psychophysics Toolbox. Spat Vis 10:433-436. CrossRef Medline

Chapman B, Stryker MP (1993) Development of orientation selectivity in ferret visual cortex and effects of deprivation. J Neurosci 13:5251-5262. Medline

Chattopadhyaya B, Di Cristo G, Higashiyama H, Knott GW, Kuhlman SJ, Welker E, Huang ZJ (2004) Experience and activity-dependent maturation of perisomatic GABAergic innervation in primary visual cortex dur- ing a postnatal critical period. J Neurosci 24:9598-9611. CrossRef Medline

Clopath C, Büsing L, Vasilaki E, Gerstner W (2010) Connectivity reflects coding: a model of voltage-based STDP with homeostasis. Nat Neurosci 13:344-352. CrossRef Medline

Demas J, Eglen SJ, Wong RO (2003) Developmental loss of synchronous spontaneous activity in the mouse retina is independent of visual experience. J Neurosci 23:2851-2860. Medline

Fiser J, Chiu C, Weliky M (2004) Small modulation of ongoing cortical dynamics by sensory input during natural vision. Nature 431:573-578. CrossRef Medline

Frégnac Y, Imbert M (1978) Early development of visual cortical cells in normal and dark-reared kittens: relationship between orientation selectivity and ocular dominance. J Physiol 278:27-44. Medline

Hofer SB, Ko H, Pichler B, Vogelstein J, Ros H, Zeng H, Lein E, Lesica NA, Mrsic-Flogel TD (2011) Differential connectivity and response dynamics of excitatory and inhibitory neurons in visual cortex. Nat Neurosci 14:1045-1052. CrossRef Medline

Hubel DH, Wiesel TN (1963) Receptive fields of cells in striate cortex of very young, visually inexperienced kittens. J Neurophysiol 26:994-1002. Medline

Katagiri H, Fagiolini M, Hensch TK (2007) Optimization of somatic inhibition at critical period onset in mouse visual cortex. Neuron 53:805-812. CrossRef Medline

Ko H, Hofer SB, Pichler B, Buchanan KA, Sjöström PJ, Mrsic-Flogel TD (2011) Functional specificity of local synaptic connections in neocortical networks. Nature 473:87-91. CrossRef Medline

Ko H, Cossell L, Baragli C, Antolik J, Clopath C, Hofer SB, Mrsic-Flogel TD (2013) The emergence of functional microcircuits in visual cortex. Nature 496:96-100. CrossRef Medline

Krug K, Akerman CJ, Thompson ID (2001) Responses of neurons in neonatal cortex and thalamus to patterned visual stimulation through the naturally closed lids. J Neurophysiol 85:1436-1443. Medline

Li Y, Fitzpatrick D, White LE (2006) The development of direction selectivity in ferret visual cortex requires early visual experience. Nat Neurosci 9:676-681. CrossRef Medline

Li Y, Lu H, Cheng PL, Ge S, Xu H, Shi SH, Dan Y (2012) Clonally related visual cortical neurons show similar stimulus feature selectivity. Nature 486:118-121. CrossRef Medline

Morales B, Choi SY, Kirkwood A (2002) Dark rearing alters the development of GABAergic transmission in visual cortex. J Neurosci 22:80848090. Medline

Ohtsuki G, Nishiyama M, Yoshida T, Murakami T, Histed M, Lois C, Ohki K (2012) Similarity of visual selectivity among clonally related neurons in visual cortex. Neuron 75:65-72. CrossRef Medline

Rochefort NL, Garaschuk O, Milos RI, Narushima M, Marandi N, Pichler B, Kovalchuk Y, Konnerth A (2009) Sparsification of neuronal activity in the visual cortex at eye opening. Proc Natl Acad Sci U S A 106:1504915054. CrossRef Medline

Rochefort NL, Narushima M, Grienberger C, Marandi N, Hill DN, Konnerth A (2011) Development of direction selectivity in mouse cortical neurons. Neuron 71:425-432. CrossRef Medline

Sarnaik R, Wang BS, Cang J (2014) Experience-dependent and independent binocular correspondence of receptive field subregions in mouse visual cortex. Cereb Cortex 24:1658-1670. CrossRef Medline

Vogelstein JT, Packer AM, Machado TA, Sippy T, Babadi B, Yuste R, Paninski L (2010) Fast nonnegative deconvolution for spike train inference from population calcium imaging. J Neurophysiol 104:3691-3704. CrossRef Medline

White LE, Coppola DM, Fitzpatrick D (2001) The contribution of sensory experience to the maturation of orientation selectivity in ferret visual cortex. Nature 411:1049-1052. CrossRef Medline

Yoshimura Y, Dantzker JL, Callaway EM (2005) Excitatory cortical neurons form fine-scale functional networks. Nature 433:868-873. CrossRef Medline

Yu YC, He S, Chen S, Fu Y, Brown KN, Yao XH, Ma J, Gao KP, Sosinsky GE, Huang K, Shi SH (2012) Preferential electrical coupling regulates neocortical lineage-dependent microcircuit assembly. Nature 486:113-117. CrossRef Medline 